\title{
The Schrödinger operator with random vector potentials
}

\author{
Kazuaki NAKANE \\ (Received March 14, 1994; Revised July 24, 1995)
}

\begin{abstract}
We consider a non-Gaussian probability measure on $\left(\mathcal{S}^{\prime}\left(\mathbf{R}^{d} ; \mathbf{R}^{d}\right), \mathcal{B}\left(\mathcal{S}^{\prime}\left(\mathbf{R}^{d} ;\right.\right.\right.$ $\left.\mathbf{R}^{d}\right)$ ) whose characteristic functional is given by Lévy-Khinchine formula. We construct a one parameter semi-group whose generator is expressed by " $(\partial-i A)^{2}$ ", $A \in \mathcal{S}^{\prime}\left(\mathbf{R}^{d}\right.$; $\mathbf{R}^{d}$ ) formally. It is also shown that the generator is self-adjoint.
\end{abstract}

Key words: electromagnetic fields, semi-group process, gauge covariance.

\section{Introduction}

Nonlinear electromagnetism is a theory of generalized random fields in four dimensional Euclidean space-time obtained by solving a system of coupled stochastic partial differential equations [AHK1, 2, AHKI, AIK1]. The fields are homogeneous with respect to the Euclidean group. It has been shown in [I, AIK3] that the fields have the sharp global Markov property. In [AIK2], relativistic time ordered functions are constructed in the model. The theory includes the usual theory of electromagnetic fields as a special case. As other special cases, it describes a class of models where the fields confine charges in Wilson's sense, and it has mild ultraviolet behavior [Ta]. Recently, in [AT], they consider a coupled theory of the confining nonlinear electromagnetic field and a charged scalar field within the quenched approximation. And they consider the propagator of matter field in approximation. The propagator is the expectation value of the resolvent kernel of the Schrödinger operator with the vector potential of the electromagnetic field. They define it as an element of $L^{p}\left(\mathcal{S}^{\prime}\left(\mathbf{R}^{d} ; \mathbf{R}^{d}\right), \mu\right)$ where $\mu$ is the probability measure which characterizes the nonlinear electromagnetic field. And they examined the asymptotic behavior of the propagator and showed that the correlation length is zero. However, they did not define the operator.

In this paper we consider the semi-group with random vector potential formally represented by $e^{t / 2(\partial-i A)^{2}}$ on $L^{2}\left(\mathbf{R}^{d}\right), d \geq 3, A \in \mathcal{S}^{\prime}\left(\mathbf{R}^{d} ; \mathbf{R}^{d}\right)$. The word "formally" reflects the fact we have not given a simultaneous definition of the operator $e^{t / 2(\partial-i A)^{2}}$ for every $A$ in the support of the measure $\mu$ on 
$\mathcal{S}^{\prime}\left(\mathbf{R}^{d} ; \mathbf{R}^{d}\right)$. We define the semi-group on $L^{2}\left(\mathbf{R}^{d}\right) \mu$-almost surely and show it is a strongly continuous symmetric contraction semi-group. Then there exists a generator of the semigroup that is self-adjoint. And we can show this operator is gauge covariant.

In section 2 , we give the definition of the measure $\mu$. In section 3 we shall show that measure $\mu$ has the property of ergodicity. In section 4 we shall show some lemmas which are needed in the following sections. In section 5 we define the semi-group $e^{t / 2(\partial-i A)^{2}}$ on $L^{2}\left(\mathbf{R}^{d}\right) \mu$-almost surely, which is measurable in the sense of [CL]. We shall show that the semi-group is a strongly-continuous symmetric contraction semi-group $\mu$-almost surely $A \in \mathcal{S}^{\prime}\left(\mathbf{R}^{d} ; \mathbf{R}^{d}\right)$ (We call it a semi-group process). Then, we can define the self-adjoint operator " $(\partial-i A)^{2}$ " $\mu$-almost surely as the generator of the semi-group. In section 6 we shall show for $\lambda \in L_{l o c}^{1}\left(\mathbf{R}^{d}\right), e^{i \lambda} e^{t / 2(\partial-i A)^{2}} e^{-i \lambda}=$ $e^{t / 2(\partial-i(A+\partial \lambda))^{2}} \mu$-almost surely. That is to say, $(\partial-i A)^{2}$ is gauge covariant. In section 7 we shall note a property of the spectrum of the generator.

\section{Notations}

In this paper we assume that $d \geq 3$. Let $\mu$ be the Borel probability measure on $\mathcal{S}^{\prime}\left(\mathbf{R}^{d} ; \mathbf{R}^{d}\right)$, the dual of the Schwartz space $\mathcal{S}\left(\mathbf{R}^{d} ; \mathbf{R}^{d}\right)$ of all $\mathbf{R}^{d}$ valued rapidly decreasing $C^{\infty}$ functions on $\mathbf{R}^{d}$, characterized by

$$
\int_{\mathcal{S}^{\prime}\left(\mathbf{R}^{d} ; \mathbf{R}^{d}\right)} e^{i\langle f, A\rangle} d \mu(A)=\exp \left(-\int_{\mathbf{R}^{d}} \psi(S f(x)) d x\right),
$$

in the sense of Minlos' theorem (see e.g. [GV]). Here $f=\left(f_{0}, f_{1}, \ldots f_{d-1}\right) \in$ $\mathcal{S}\left(\mathbf{R}^{d} ; \mathbf{R}^{d}\right)$, and $S f$ is defined by

$$
(S f)_{\nu}(x)=\sum_{\rho=0}^{d-1} \int_{\mathbf{R}^{d}} S_{\nu \rho}(x-y) f_{\rho}(y) d y,
$$

with

$$
\begin{aligned}
S_{0 \nu}(x-y) & =\partial_{\nu}(-\Delta)^{-1}(x, y)=-\frac{\Gamma(d / 2)}{2 \pi^{d / 2}} \frac{x_{\nu}-y_{\nu}}{|x-y|^{d}} \\
S_{j 0}(x-y) & =-\partial_{j}(-\Delta)^{-1}(x, y)=\frac{\Gamma(d / 2)}{2 \pi^{d / 2}} \frac{x_{j}-y_{j}}{|x-y|^{d}} \\
S_{i j}(x-y) & =\left(\delta_{i j} \partial_{0}+\sum_{k=1}^{d-1} a_{i j k} \partial_{k}\right)(-\Delta)^{-1}(x, y) \\
& =-\frac{\Gamma(d / 2)}{2 \pi^{d / 2}} \frac{\left(x_{0}-y_{0}\right) \delta_{i j}+\sum_{k=1}^{d-1} a_{i j k}\left(x_{k}-y_{k}\right)}{|x-y|^{d}}
\end{aligned}
$$


for $x=\left(x_{0}, \cdots, x_{d-1}\right), 0 \leq \nu \leq d-1$ and $1 \leq i, j \leq d-1$. Here $\delta_{i j}$ is Kronecker's delta and $a_{i j k}$ is a real constant. $\psi$ is a function on $\mathbf{R}^{d}$, represented by the Lévy-Khinchine formula

$$
\psi(\lambda)=\int_{\mathbf{R}^{d}}\left(1-e^{i \alpha \cdot \lambda}+\frac{i \alpha \cdot \lambda}{1+|\alpha|^{2}}\right) d \nu(\alpha)+\frac{1}{2}\langle\lambda, \mathbf{A} \lambda\rangle
$$

where $\mathbf{A}$ is a non-negative definite $d \times d$ matrix and $\nu$ is a measure on $\mathbf{R}^{d}$ such that $\nu(\{0\})=0, \int_{\mathbf{R}^{d}}\left(|\alpha|^{2} \wedge 1\right) d \nu(\alpha)<\infty$.

In this paper we assume that $\psi$ satisfies the following conditions:

(A.1): The function $\psi$ is continuous, non-negative and bounded, i.e. there exists a constant $c_{0}$ such that

$$
0 \leq \psi \leq c_{0}
$$

(A.2): There exist constants $c_{1}>0$ and $\frac{d}{d-1}<\eta \leq 2$ such that

$$
|\psi(\lambda)| \leq c_{1}|\lambda|^{\eta}, \quad \text { for } \quad|\lambda| \leq 1 .
$$

The conditions (A.1) (A.2) are slightly weak compared with those in $[\mathrm{AT}]$.

Remark 2.1. Under (A.1), A must be 0 in the representation (2.4).

Remark 2.2. The function $\psi$ which satisfies these conditions may grow as $|\lambda|^{\eta}$ for large $|\lambda|$; i.e. there exists a constant $c>0$,

$$
\psi(\lambda) \leq c|\lambda|^{\eta}, \quad \text { for } \quad \lambda \in \mathbf{R}^{d} .
$$

We give here two examples of $\psi$ which satisfies the above conditions for the case $d=4$.

Example 2.3. The function

$$
\psi(\lambda)=\frac{|\lambda|^{\eta}}{1+|\lambda|^{\eta}} \quad \lambda \in \mathbf{R}^{4}
$$

satisfies (A.1), (A.2).

Example 2.4. Let $\alpha=\left(\alpha_{0}, \alpha^{\prime}\right)=\left(\alpha_{0}, \alpha_{1}, \alpha_{2}, \alpha_{3}\right) \in \mathbf{R}^{4}$. The function $\psi$ which is defined by the equation (2.4) with the matrix $\mathbf{A}=0$ and measure 


$$
\begin{aligned}
& \nu(\alpha)=\delta\left(\alpha_{0}\right) \nu^{\prime}\left(\alpha^{\prime}\right), \\
& \quad d \nu^{\prime}\left(\alpha^{\prime}\right)=\frac{H\left(\left|\alpha^{\prime}\right|-1\right)}{\left|\alpha^{\prime}\right|^{3+\eta}} d \alpha^{\prime},
\end{aligned}
$$

where $d \alpha^{\prime}$ is the Lebesgue measure on $\mathbf{R}^{3}$ and $H$ is the Heviside function satisfies (A.1), (A.2).

The proofs are in $[\mathrm{Ta}]$.

Let $I: \mathbf{R} \rightarrow[0,1]$ be a $C_{0}^{\infty}$ function such that

$$
I(s)= \begin{cases}1, & |s| \leq \frac{1}{4} \\ 0, & |s| \geq \frac{1}{2}\end{cases}
$$

and set

$$
I_{l}(s)=\left\{\begin{array}{ll}
1, & |s| \leq l-1, \\
I(|s|-l+1), & l-1 \leq|s| \leq l, \\
0, & |s| \geq l,
\end{array} \quad \text { for } \quad l \in \mathbf{N} .\right.
$$

Then the function

$$
\rho_{\varepsilon}(x)=\frac{1}{\varepsilon^{d}\|I\|_{L^{1}}^{d}} \prod_{\nu=0}^{d-1} I\left(\frac{x_{\nu}}{\varepsilon}\right) \quad(\varepsilon>0)
$$

satisfies

$$
\begin{aligned}
& \rho_{\varepsilon} \in \mathcal{D}\left(\mathbf{R}^{d}\right), \quad \operatorname{supp} \rho_{\varepsilon} \subset\left\{x \in \mathbf{R}^{d}|| x \mid=\left(\sum_{\nu=0}^{d-1}\left|x_{\nu}\right|^{2}\right)^{1 / 2} \leq \frac{\sqrt{d}}{2} \varepsilon\right\}, \\
& \int_{\mathbf{R}^{d}} \rho_{\varepsilon}(x) d x=1, \quad \rho_{\varepsilon} \geq 0,
\end{aligned}
$$

and hence

$$
\lim _{\varepsilon \rightarrow 0} \rho_{\varepsilon}=\delta, \quad \text { in } \mathcal{S}^{\prime}\left(\mathbf{R}^{d}\right),
$$

where $\|\cdot\|_{L^{1}}$ is the norm of $L^{1}\left(\mathbf{R}^{d}, d x\right)$ with Lebesgue measure $d x$ and $\mathcal{D}\left(\mathbf{R}^{d}\right)$ is a family of $C^{\infty}$ functions with compact support. The function

$$
w_{l}(x)=\prod_{\nu=0}^{d-1} I_{l}\left(x_{\nu}\right)
$$


satisfies

$$
w_{l} \in \mathcal{D}\left(\mathbf{R}^{d}\right), \quad 0 \leq w_{l} \leq 1,
$$

and

$$
w_{l} \uparrow 1 \quad \text { as } \quad l \rightarrow \infty .
$$

For each $A \in \mathcal{S}^{\prime}\left(\mathbf{R}^{d} ; \mathbf{R}^{d}\right)$, we define $A^{n}(n \in \mathbf{N})$ by

$$
A_{\nu}^{n}(x)=w_{n}(x)\left\langle\rho_{1 / n}(x-\cdot), A_{\nu}\right\rangle, \quad \nu=0,1,2, \ldots, d-1 .
$$

Obviously $A^{n} \in \mathcal{D}\left(\mathbf{R}^{d} ; \mathbf{R}^{d}\right)$ and

$$
\lim _{n \rightarrow \infty} A^{n}=A, \quad \text { in } \quad \mathcal{S}^{\prime}\left(\mathbf{R}^{d} ; \mathbf{R}^{d}\right) .
$$

Theorem 2.5 (Feynman-Kac-Itô) Let $A \in \mathcal{D}\left(\mathbf{R}^{d} ; \mathbf{R}^{d}\right)$. For any $f \in$ $\mathcal{D}\left(\mathbf{R}^{d}\right)$,

$$
\begin{array}{r}
e^{t / 2(\partial-i A)^{2}} f(x)=E\left[f ( x + b ( t ) ) \operatorname { e x p } \left(-i \sum_{\nu=0}^{d-1} \int_{0}^{t} A_{\nu}(x+b(s)) d b_{\nu}(s)\right.\right. \\
\left.\left.-\frac{i}{2} \sum_{\nu=0}^{d-1} \int_{0}^{t} \partial_{\nu} A_{\nu}(x+b(s)) d s\right)\right], \quad(2.12)
\end{array}
$$

where $E$ denotes the expectation with respect to a d dimensional standard Brownian motion $\left(C\left([0, \infty) ; \mathbf{R}^{d}\right) \quad \mathcal{B}\left(C\left([0, \infty) ; \mathbf{R}^{d}\right)\right), \quad\{b(s)\}_{s \in[0, \infty)}\right.$, $\left.\left\{\mathcal{F}_{s}^{b}\right\}_{s \in[0, \infty)}, P\right)$.

Proof. See [Si].

The operators $\left\{e^{t / 2(\partial-i A)^{2}}\right\}_{t \geq 0}$ on $L^{2}\left(\mathbf{R}^{d}\right)$ form a strongly continuous symmetric contraction semi-group. (See $[\mathrm{Si}],[\mathrm{F}]$ ).

Lemma 2.6 For any $t>0, f \in \mathcal{D}\left(\mathbf{R}^{d} \times \mathbf{R}^{d}\right)$ and $x \in \mathbf{R}^{d}$, we can take a suitable version of $\int_{0}^{t} f(x+b(s), \cdot) d b_{\nu}(s)$ such that

$$
\int_{0}^{t} f(x+b(s), \cdot) d b_{\nu}(s) \in \mathcal{D}\left(\mathbf{R}^{d}\right), \quad \text { P-a.s. }
$$

and

$$
\begin{array}{r}
\left\langle\int_{0}^{t} f(x+b(s), \cdot) d b_{\nu}(s), \varphi\right\rangle=\int_{0}^{t}\langle f(x+b(s), \cdot), \varphi\rangle d b_{\nu}(s), \\
P-a . s .
\end{array}
$$


holds for every $\varphi \in \mathcal{D}^{\prime}\left(\mathbf{R}^{d}\right), 0 \leq \nu \leq d-1$. The equality

$$
\left\langle\int_{0}^{t} g(x+b(s), \cdot) d s, \varphi\right\rangle=\int_{0}^{t}\langle g(x+b(s), \cdot), \varphi\rangle d s, \quad P-a . s .
$$

also holds for any $g \in \mathcal{D}\left(\mathbf{R}^{d} \times \mathbf{R}^{d}\right)$, and $\varphi \in \mathcal{D}^{\prime}\left(\mathbf{R}^{d}\right)$.

Proof. We can show this lemma in the same way as in [AT Lemma 2]. So we omit here the proof.

\section{Ergodicity}

In this section, we shall show the probability measure $\mu$ on $\left(\mathcal{S}^{\prime}\left(\mathbf{R}^{d} ; \mathbf{R}^{d}\right)\right.$, $\left.\mathcal{B}\left(\mathcal{S}^{\prime}\left(\mathbf{R}^{d} ; \mathbf{R}^{d}\right)\right)\right)$ defined in section 2 has the ergodic property. Here we assume that $\varphi$ is a function on $\mathbf{R}^{d}$, represented by the Lévy-Khinchine formula (2.3) and satisfies the condition (A). We define the transformations $\left\{\theta_{z}\right\}_{z \in \mathbf{R}^{d}}$ on $\mathcal{S}^{\prime}\left(\mathbf{R}^{d} ; \mathbf{R}^{d}\right)$ by

$$
\theta_{z} A \equiv A(\cdot-z), \quad A \in \mathcal{S}^{\prime}\left(\mathbf{R}^{d} ; \mathbf{R}^{d}\right) .
$$

It is obvious that

$$
\theta_{0}=\text { Identity, } \theta_{x} \circ \theta_{y}=\theta_{x+y}, \quad x, y \in \mathbf{R}^{d} .
$$

In the following, we shall show the family of transformations $\left\{\theta_{z}\right\}_{z \in \mathbf{R}^{d}}$ is a family of measure preserving transformations on $\mathcal{S}^{\prime}\left(\mathbf{R}^{d} ; \mathbf{R}^{d}\right)$ and it is an ergodic family.

Lemma 3.1 $\left\{\theta_{z}\right\}_{z \in \mathbf{R}^{d}}$ is a family of measure preserving transformations on $\mathcal{S}^{\prime}\left(\mathbf{R}^{d} ; \mathbf{R}^{d}\right)$; i.e.

$$
\mu(B)=\mu\left(\theta_{z} B\right), \quad B \in \mathcal{B}\left(\mathcal{S}^{\prime}\left(\mathbf{R}^{d} ; \mathbf{R}^{d}\right)\right) .
$$

Proof. To show this lemma, it is sufficient that the characteristic functional defined by (2.1) is invariant under this transformation. By the definition of the characteristic functional,

$$
\begin{aligned}
\int_{\mathcal{S}^{\prime}\left(\mathbf{R}^{d} ; \mathbf{R}^{d}\right)} \exp i\left\langle f, \theta_{z} A\right\rangle d \mu & =\int_{\mathcal{S}^{\prime}\left(\mathbf{R}^{d} ; \mathbf{R}^{d}\right)} \exp i\langle f(\cdot+z), A\rangle d \mu \\
& =\exp \left(-\int_{\mathbf{R}^{d}} \varphi(S f(x+z)) d x\right) \\
& =\exp \left(-\int_{\mathbf{R}^{d}} \varphi(S f(x)) d x\right)
\end{aligned}
$$




$$
=\int_{\mathcal{S}^{\prime}\left(\mathbf{R}^{d} ; \mathbf{R}^{d}\right)} \exp i\langle f, A\rangle d \mu .
$$

Moreover, we can show that $\left\{\theta_{z}\right\}_{z \in \mathbf{R}^{d}}$ is an ergodic family.

Theorem 3.2 The family of measure preserving transformations $\left\{\theta_{z}\right\}_{z \in \mathbf{R}^{d}}$ on $\mathcal{S}^{\prime}\left(\mathbf{R}^{d} ; \mathbf{R}^{d}\right)$ is an ergodic family.

To show this theorem, we shall show the measure $\mu$ satisfies the mixing property. Before the proof of Theorem 3.2, we shall show two lemmas.

Lemma 3.3 Suppose that $V$ is a finite dimensional vector space over $\mathbf{R}$ and $\mathcal{A}$ is a $\mathbf{C}$-module functions of $\mathcal{S}^{\prime}\left(\mathbf{R}^{d} ; V\right)$ generated by $\left\{e^{i\langle\xi, \cdot\rangle} ; \xi \in\right.$ $\left.\mathcal{D}\left(\mathbf{R}^{d} ; V\right)\right\}$. Then $\mathcal{A}$ is a $\mathbf{C}$-algebra. If $\mu$ is a probability measure on $\mathcal{S}^{\prime}\left(\mathbf{R}^{d} ; V\right)$ then $\mathcal{A}$ is dense in $L^{2}\left(\mathcal{S}^{\prime}\left(\mathbf{R}^{d} ; V\right), \mu\right)$.

Proof. We can show this lemma by slightly modifying the proof of Theorem $4.1[\mathrm{Hi}]$.

Lemma 3.4 Suppose that $V$ is a finite dimensional vector space over $\mathbf{R}$ and $\varphi: V \rightarrow \mathbf{R}$ is a continuous function such that $\varphi(\lambda)=O\left(|\lambda|^{\eta}\right)$ as $\lambda \rightarrow 0$ for some $\eta \in[1, \infty)$. If $f, g: \mathbf{R}^{d} \rightarrow V$ are continuous functions vanishing at $\infty$ and $|f|^{\eta},|g|^{\eta}$ are integrable with respect to the Lebesgue measure, then

$$
\lim _{x \rightarrow \infty} \int_{\mathbf{R}^{d}} \varphi(f(x+y)+g(y)) d y=\int_{\mathbf{R}^{d}} \varphi(f(y)) d y+\int_{\mathbf{R}^{d}} \varphi(g(y)) d y .
$$

Proof. $\quad$ Because of the assumption, $\varphi(f(x+\cdot)+g(\cdot)), \varphi(f(x+\cdot))$ and $\varphi(g(\cdot))$ are integrable. For any $\varepsilon>0$, there exists $N>0$ such that for any $x \in \mathbf{R}$,

$$
\int_{\left(B_{N}(0) \cup B_{N}(-x)\right)^{c}}|\varphi(f(x+y)+g(y))-\varphi(f(x+y))-\varphi(g(y))| d y<\varepsilon,
$$

where $B_{N}(\cdot)$ is a closed ball with radius $N$ and center $\cdot$ We take $x$ sufficiently large and we can assume that $B_{N}(0) \cap B_{N}(-x)=\emptyset$. We have

$$
\begin{gathered}
\int_{\left(B_{N}(0) \cup B_{N}(-x)\right)}|\varphi(f(x+y)+g(y))-\varphi(f(x+y))-\varphi(g(y))| d y \\
=\int_{B_{N}(0)}|\varphi(f(x+y)+g(y))-\varphi(f(x+y))-\varphi(g(y))| d y
\end{gathered}
$$




$$
\begin{aligned}
& +\int_{B_{N}(-x)}|\varphi(f(x+y)+g(y))-\varphi(f(x+y))-\varphi(g(y))| d y \\
\leq & \left(\max _{y \in B_{N}(0)}|\varphi(f(x+y))|+\max _{y \in B_{N}(-x)}|\varphi(g(y))|\right) \operatorname{Vol} B_{N}(0) \\
& +\int_{B_{N}(0)}|\varphi(f(x+y)+g(y))-\varphi(g(y))| d y \\
& +\int_{B_{N}(-x)}|\varphi(f(x+y)+g(y))-\varphi(f(x+y))| d y .
\end{aligned}
$$

Then we get

$$
\begin{aligned}
(3.1)= & \left(\max _{y \in B_{N}(0)}|\varphi(f(x+y))|+\max _{y \in B_{N}(0)}|\varphi(g(y-x))|\right) \operatorname{Vol} B_{N}(0) \\
& +\int_{B_{N}(0)}|\varphi(f(x+y)+g(y))-\varphi(g(y))| d y \\
& +\int_{B_{N}(0)}|\varphi(f(y)+g(y-x))-\varphi(f(y))| d y
\end{aligned}
$$

By the assumption of $\varphi, f$ and $g$, we have

$$
\begin{aligned}
& \max _{y \in B_{N}(0)}|\varphi(f(x+y)+g(y))-\varphi(g(y))| \rightarrow 0, \\
& \max _{y \in B_{N}(0)}|\varphi(f(y)+g(y-x))-\varphi(f(y))| \rightarrow 0, \quad \text { as } \quad|x| \rightarrow \infty .
\end{aligned}
$$

So we can conclude that (3.2) tends to 0 as $x \rightarrow \infty$.

Proof of Theorem 3.2. We only show, for any $\xi_{1}, \xi_{2} \in \mathcal{D}\left(\mathbf{R}^{d} ; \mathbf{R}^{d}\right)$,

$$
\begin{aligned}
& \lim _{z \rightarrow \infty} \int_{\mathcal{S}^{\prime}\left(\mathbf{R}^{d} ; \mathbf{R}^{d}\right)} e^{i\left\langle\xi_{1}+\theta_{z} \xi_{2}, \cdot\right\rangle} d \mu \\
& \quad=\int_{\mathcal{S}^{\prime}\left(\mathbf{R}^{d} ; \mathbf{R}^{d}\right)} e^{i\left\langle\xi_{1}, \cdot\right\rangle} d \mu \int_{\mathcal{S}^{\prime}\left(\mathbf{R}^{d} ; \mathbf{R}^{d}\right)} e^{i\left\langle\xi_{2}, \cdot\right\rangle} d \mu .
\end{aligned}
$$

We have

$$
\begin{aligned}
\int_{\mathcal{S}^{\prime}\left(\mathbf{R}^{d} ; \mathbf{R}^{d}\right)} e^{i\left\langle\xi_{1}+\theta_{z} \xi_{2}, \cdot\right\rangle} d \mu & =\exp \left(-\int_{\mathbf{R}^{d}} \varphi\left(S\left(\xi_{1}+\theta_{z} \xi_{2}\right)\right) d x\right) \\
\int_{\mathcal{S}^{\prime}\left(\mathbf{R}^{d} ; \mathbf{R}^{d}\right)} e^{i\left\langle\xi_{1}, \cdot\right\rangle} d \mu & =\exp \left(-\int_{\mathbf{R}^{d}} \varphi\left(S\left(\xi_{1}\right)\right) d x\right) \\
\int_{\mathcal{S}^{\prime}\left(\mathbf{R}^{d} ; \mathbf{R}^{d}\right)} e^{i\left\langle\xi_{2}, \cdot\right\rangle} d \mu & =\exp \left(-\int_{\mathbf{R}^{d}} \varphi\left(S\left(\xi_{2}\right)\right) d x\right) .
\end{aligned}
$$

Since $S\left(\xi_{1}\right)$ and $S\left(\xi_{2}\right)$ are continuous and vanishing at $\infty,\left|S\left(\xi_{1}\right)\right|^{\eta}$ and 
$\left|S\left(\xi_{2}\right)\right|^{\eta}$ are integrable, then, by Lemma 3.4, we get (3.3).

\section{Preliminaries}

In this section, we will prove some important lemmas. For any $t_{0}>0$, we set $\mathcal{T}=\left(0, t_{0}\right]$. We set for any $t \in \mathcal{T}$,

$$
\begin{aligned}
T_{t}^{A, n} f(x) \equiv & e^{t / 2\left(\partial-i A^{n}\right)^{2}} f(x) \\
= & E\left[f ( x + b ( t ) ) \operatorname { e x p } \left(-i \sum_{\nu=0}^{d-1} \int_{0}^{t} A_{\nu}^{n}(x+b(s)) d b_{\nu}(s)\right.\right. \\
& \left.\left.\quad-\frac{i}{2} \sum_{\nu=0}^{d-1} \int_{0}^{t} \partial_{\nu} A_{\nu}^{n}(x+b(s)) d s\right)\right], \text { for } f \in \mathcal{D}\left(\mathbf{R}^{d}\right),
\end{aligned}
$$

where $A^{n} \in \mathcal{D}\left(\mathbf{R}^{d} ; \mathbf{R}^{d}\right)$ in $(2.10)$.

Lemma 4.1 For any $d>2$ and $T>0$, we set

$$
f(s, x)=|x|^{2-d}(2 T-2 s)^{(d-4) / 2} \exp \left(\frac{-|x|^{2}}{2(T-s)}\right) .
$$

Then $f(s, x)$ is a solution of the partial differential equation

$$
\frac{\partial f}{\partial s}+\frac{1}{2} \Delta f=0
$$

at $(s, x) \in(-\infty, T) \times \mathbf{R}^{d} \backslash\{0\}$. Moreover, $f(t, x) \geq f(t, y) \Leftrightarrow|x| \leq|y|$ for each $t<T$.

Proof. We can show this by a direct calculation.

Lemma 4.2 Let $0<t<T$ and $\varepsilon>0$. Then, for any $d>2$,

$$
E\left[\operatorname{Vol}\left(\left\{z\left|\inf _{s \in[0, t]}\right| b(s)-z \mid \leq \varepsilon\right\}\right)\right] \leq \varepsilon^{d-2} \cdot K(d, t, T, \varepsilon),
$$

where

$$
\begin{gathered}
K(d, t, T, \varepsilon)=\frac{\pi^{d / 2}}{\Gamma(d / 2+1)} \varepsilon^{2}+\frac{2 \pi^{d / 2}}{\Gamma(d / 2)}\left(\frac{T}{T-t}\right)^{(d-4) / 2 \vee 0} T \\
\exp \left(\frac{\varepsilon^{2}}{2(T-t)}-\frac{\varepsilon^{2}}{2 T}\right)
\end{gathered}
$$


Proof. Let $\varepsilon>0$ and $0<t<T$. We define a stopping time $\tau_{\varepsilon}(a)$ by

$$
\tau_{\varepsilon}(a) \equiv \inf \{s \in[0, \infty)|| b(s)+a \mid=\varepsilon\} \wedge t
$$

for $a \in \mathbf{R}^{d} \backslash\{0\}$ and $\varepsilon<|a|$. Let $X(s)$ be the process

$$
X(s)=b\left(s \wedge \tau_{\varepsilon}(a)\right)+a=\int_{0}^{s} I_{u \leq \tau_{\varepsilon}(a)} d b(u)+a .
$$

By Lemma 4.1, the process $f\left(s \wedge \tau_{\varepsilon}(a), X(s)\right)$ is a martingale. In fact

$$
\begin{aligned}
& f(s\left.\wedge \tau_{\varepsilon}(a), X(s)\right) \\
&=f(0, a)+\sum_{\nu=0}^{d-1} \int_{0}^{s} I_{u \leq \tau_{\varepsilon}(a)} \frac{\partial f}{\partial x_{\nu}}(u, X(u)) d X_{\nu}(u) \\
& \quad+\int_{0}^{s} I_{u \leq \tau_{\varepsilon}(a)}\left\{\frac{\partial f}{\partial u}(u, X(u))+\frac{1}{2} \Delta f(u, X(u))\right\} d u \\
&=f(0, a)+\sum_{\nu=0}^{d-1} \int_{0}^{s \wedge \tau_{\varepsilon}(a)} \frac{\partial f}{\partial x_{\nu}}(u, X(u)) d X_{\nu}(u) .
\end{aligned}
$$

And by the Doob martingale inequality, we have

$$
\begin{aligned}
& P\left[\left\{\omega\left|\inf _{s \in[0, t]}\right| X(s, \omega) \mid \leq \varepsilon\right\}\right] \\
& \leq P\left[\left\{\omega \mid \sup _{s \in[0, t]} f\left(s \wedge \tau_{\varepsilon}(a), X(s, \omega)\right)\right.\right. \\
& \left.\left.\quad \geq \varepsilon^{2-d} \min _{s \in[0, t]}\left\{(2 T-2 s)^{(d-4) / 2}\right\} \exp \frac{-\varepsilon^{2}}{2(T-t)}\right\}\right] \\
& \leq \varepsilon^{d-2} \max _{s \in[0, t]}\left\{(2 T-2 s)^{(4-d) / 2}\right\} \exp \frac{\varepsilon^{2}}{2(T-t)} E\left[f\left(t \wedge \tau_{\varepsilon}(a), X(t, \omega)\right)\right] \\
& =\varepsilon^{d-2} \max _{s \in[0, t]}\left\{(2 T-2 s)^{(4-d) / 2}\right\} \exp \frac{\varepsilon^{2}}{2(T-t)} E[f(0, X(0, \omega))] \\
& =\left(\frac{\varepsilon}{|a|}\right)^{d-2}(2 T)^{(d-4) / 2} \max _{s \in[0, t]}\left\{(2 T-2 s)^{(4-d) / 2}\right\} \\
& \quad \exp \left(\frac{\varepsilon^{2}}{2(T-t)}-\frac{|a|^{2}}{2 T}\right) .
\end{aligned}
$$

The estimate (4.5) leads to

$$
E\left[\operatorname{Vol}\left\{z\left|\inf _{s \in[0, t]}\right| b(s)-z \mid \leq \varepsilon\right\}\right]
$$




$$
\begin{aligned}
& =\int_{\mathbf{R}^{d}} P\left[\left\{\omega\left|\inf _{s \in[0, t]}\right| b(s)-z \mid \leq \varepsilon\right\}\right] d z \\
& =\int_{\mathbf{R}^{d}} \chi_{\{|z| \leq \varepsilon\}}+\chi_{\{|z|>\varepsilon\}} P\left[\left\{\omega\left|\inf _{s \in[0, t]}\right| b(s)-z \mid \leq \varepsilon\right\}\right] d z \\
& \leq \frac{\pi^{d / 2}}{\Gamma(d / 2+1)} \varepsilon^{d}+\int_{\varepsilon}^{\infty}\left(\frac{\varepsilon}{r}\right)^{d-2}(2 T)^{(d-4) / 2} \max _{s \in[0, t]}\left\{(2 T-2 s)^{(4-d) / 2}\right\} \\
& =\frac{\pi^{d / 2}}{\Gamma(d / 2+1)} \varepsilon^{d}+\frac{2 \pi^{d / 2}}{\Gamma(d / 2)} \varepsilon^{d-2}(2 T)^{(d-4) / 2} \max _{s \in[0, t]}\left\{(2 T-2 s)^{(4-d) / 2}\right\} \\
& \cdot T \cdot \exp \left(\frac{\varepsilon^{2}}{2(T-t)}-\frac{\varepsilon^{2}}{2 T}\right),
\end{aligned}
$$

where $\chi_{K}$ is the indicator function of the set $K$. We readily conclude (4.4).

For each $f \in \mathcal{D}\left(\mathbf{R}^{d}\right)$ and $x \in \mathbf{R}^{d}$, it is obvious that $T_{t}^{A, n} f(x)$ is $\mathcal{B}\left(\mathcal{S}^{\prime}\left(\mathbf{R}^{d} ; \mathbf{R}^{d}\right)\right)$ measurable $[\mathrm{AT}]$. By using Lemma 2.5 , we have for any $t \in \mathcal{T}$,

$$
\begin{aligned}
& F_{m, n}^{t}(x) \equiv\left\|T_{t}^{A, m} f(x)-T_{t}^{A, n} f(x)\right\|_{L^{2}\left(\mathcal{S}^{\prime}\left(\mathbf{R}^{d} ; \mathbf{R}^{d}\right)\right)}^{2} E[|f(x+b(t))| \\
& \leq \int_{\mathcal{S}^{\prime}\left(\mathbf{R}^{d} ; \mathbf{R}^{d}\right)} \\
& \times \mid 1-\exp \left\{-i\left(\sum_{\nu=0}^{d-1} \int_{0}^{t}\left(A_{\nu}^{m}-A_{\nu}^{n}\right) d b_{\nu}(s)\right.\right. \\
& \left.\left.\quad+\frac{1}{2} \sum_{\nu=0}^{d-1} \int_{0}^{t}\left(\partial_{\nu} A_{\nu}^{m}-\partial_{\nu} A_{\nu}^{n}\right) d s\right)\right\}\left.\right|^{2} d \mu \\
& \leq \int_{\mathcal{S}^{\prime}\left(\mathbf{R}^{d} ; \mathbf{R}^{d}\right)} E\left[|f(x+b(t))|^{2}\right. \\
& \times 2\left\{1-\cos \left(\sum _ { \nu = 0 } ^ { d - 1 } \left\langle\int_{0}^{t} f_{m, n}(x+b(s), \cdot) d b_{\nu}(s)\right.\right.\right. \\
& \left.\left.\left.\left.\quad+\int_{0}^{t} \frac{1}{2} g_{m, n ; \nu}(x+b(s), \cdot) d s, A_{\nu}\right\rangle\right)\right\}\right] d \mu
\end{aligned}
$$

where

$$
f_{m, n}(x, y) \equiv w_{m}(x) \rho_{1 / m}(x-y)-w_{n}(x) \rho_{1 / n}(x-y)
$$




$$
g_{m, n ; \nu}(x, y) \equiv \frac{\partial}{\partial x_{\nu}} f_{m, n}(x, y)
$$

By (2.4) and Fubini's theorem, we have

$$
F_{n, m}^{t}(x) \leq 2 E\left[|f(x+b(t))|^{2}\left(1-\exp \left(-\int_{\mathbf{R}^{d}} \psi_{m, n}(t, z, x) d z\right)\right)\right],
$$

where

$$
\begin{aligned}
\psi_{m, n}(t, z, x)= & \psi\left(\sum_{\nu=0}^{d-1} \int_{0}^{t} d b_{\nu}(s) \int_{\mathbf{R}^{d}} d v S_{\cdot \nu}(z-v) f_{m, n}(x+b(s), v)\right. \\
& \left.+\frac{1}{2} \sum_{\nu=0}^{d-1} \int_{0}^{t} d s \int_{\mathbf{R}^{d}} d v S_{\cdot \nu}(z-v) g_{m, n ; \nu}(x+b(s), v)\right) .
\end{aligned}
$$

Lemma 4.3 For each $x \in \mathbf{R}^{d}, E\left[\exp \left(-\int_{\mathbf{R}^{d}} \psi_{m, n}(t, z, x) d z\right)\right]$ converges to 1 uniformly in $t \in \mathcal{T}$ as $m, n \rightarrow \infty$.

Proof. By Jensen's inequality,

$$
\begin{aligned}
0 & \leq E\left[1-\exp \left(-\int_{\mathbf{R}^{d}} \psi_{m, n}(t, z, x) d z\right)\right] \\
& \leq 1-\exp \left(-E\left[\int_{\mathbf{R}^{d}} \psi_{m, n}(t, z, x) d z\right]\right) .
\end{aligned}
$$

So it is sufficient to show that

$$
G_{m, n}(x) \equiv \sup _{t \in \mathcal{T}} E\left[\int_{\mathbf{R}^{d}} \psi_{m, n}(t, z, x) d z\right] \rightarrow 0 \quad \text { as } \quad n, m \rightarrow \infty .
$$

Put

$$
V(t, x+b, \varepsilon) \equiv\left\{z \in \mathbf{R}^{d}\left|\inf _{s \in[0, t]}\right| x+b(s)-z \mid \leq \varepsilon\right\},
$$

then by $(\mathrm{A})$,

$$
\begin{aligned}
G_{m, n}(x) \leq & \sup _{t \in \mathcal{T}} E\left[\int_{V(t, x+b, \varepsilon)} \psi_{m, n}(t, z, x) d z\right] \\
& \quad+\sup _{t \in \mathcal{T}} E\left[\int_{V^{c}(t, x+b, \varepsilon)} \psi_{m, n}(t, z, x) d z\right] \\
\leq & \sup _{t \in \mathcal{T}} c_{0} E[\operatorname{Vol} V(t, x+b, \varepsilon)] \\
& +\sup _{t \in \mathcal{T}} E\left[\int_{V^{c}(t, x+b, \varepsilon)} \psi_{m, n}(t, z, x) d z\right]
\end{aligned}
$$




$$
\begin{aligned}
= & c_{0} E\left[\operatorname{Vol} V\left(t_{0}, x+b, \varepsilon\right)\right] \\
& +\sup _{t \in \mathcal{T}} E\left[\int_{V^{c}(t, x+b, \varepsilon)} \psi_{m, n}(t, z, x) d z\right],
\end{aligned}
$$

because $V(t, x+b, \varepsilon)$ is increasing in $t$. By Lemma 4.2, the first term of (4.8) is dominated as follows:

$$
\begin{aligned}
E\left[\operatorname{Vol} V\left(t_{0}, x+b, \varepsilon\right)\right] & =\int_{\mathbf{R}^{d}} P\left[\left\{\omega\left|\inf _{s \in\left[0, t_{0}\right]}\right| x+b(s)-z \mid \leq \varepsilon\right\}\right] d z \\
& \leq \varepsilon^{d-2} \cdot K\left(d, t_{0}, t_{0}+1, \varepsilon\right) .
\end{aligned}
$$

We shall show in the following the second term tends to 0 as $n, m \rightarrow \infty$ for arbitrary but fixed $\varepsilon>0$. We may assume that $n$ and $m$ are so large that $1 / n, 1 / m<\varepsilon / \sqrt{d}$ hold. By (2.6), we have

$$
\begin{aligned}
& \sup _{t \in \mathcal{T}} E\left[\int_{V^{c}(t, x+b, \varepsilon)} \psi_{m, n}(t, z, x) d z\right] \\
& \leq \sup _{t \in \mathcal{T}} c_{1} E\left[\int _ { \mathbf { R } ^ { d } } \chi _ { V ^ { c } ( t , x + b , \varepsilon ) } ( z ) d z \left(\sum_{j=1}^{d} \mid \sum_{\nu=0}^{d-1} \int_{0}^{t} d b_{\nu}(s)\right.\right. \\
& \quad \int_{\mathbf{R}^{d}} d v S_{j \nu}(z-v) f_{m, n}(x+b(s), v) \\
& \left.\left.+\left.\frac{1}{2} \sum_{\nu=0}^{d-1} \int_{0}^{t} d s \int_{\mathbf{R}^{d}} d v S_{j \nu}(z-v) g_{m, n ; \nu}(x+b(s), v)\right|^{2}\right)^{\eta / 2}\right] .
\end{aligned}
$$

Since the following inequality

$$
\begin{aligned}
& E\left[\left(\sum_{j=1}^{d}\left|\sum_{\nu=0}^{d-1} a_{j \nu}(\omega)\right|^{2}\right)^{\eta / 2}\right] \leq E\left[\left(d \sum_{j, \nu} a_{j \nu}^{2}\right)^{\eta / 2}\right] \\
& \leq d^{\eta / 2}\left(\sum_{j, \nu} E\left[a_{j \nu}^{2}\right]\right)^{\eta / 2} \leq d^{\eta / 2} \sum_{j, \nu}\left(E\left[a_{j \nu}^{2}\right]\right)^{\eta / 2}
\end{aligned}
$$

holds for real $a_{j \nu}$ 's, which is a consequence of Jensen's inequality (recall $\eta / 2 \leq 1)$. We get

$$
\begin{aligned}
& \sup _{t \in \mathcal{T}} E\left[\int_{V^{c}(t, x+b, \varepsilon)} \psi_{m, n}(t, z, x) d z\right] \\
& \leq \sup _{t \in \mathcal{T}} d^{\eta / 2} c_{1} \sum_{j, \nu} \int_{\mathbf{R}^{d}} d z\left(E \left[\chi_{V^{c}(t, x+b, \varepsilon)}(z)\right.\right. \\
& \quad \mid \int_{0}^{t} d b_{\nu}(s) \int_{\mathbf{R}^{d}} d v S_{j \nu}(z-v) f_{m, n}(x+b(s), v)
\end{aligned}
$$




$$
\begin{aligned}
& \left.\left.+\left.\frac{1}{2} \int_{0}^{t} d s \int_{\mathbf{R}^{d}} d v S_{j \nu}(z-v) g_{m, n ; \nu}(x+b(s), v)\right|^{2}\right]\right)^{\eta / 2} \\
& \leq \sup _{t \in \mathcal{T}} d^{\eta / 2} c_{1} \sum_{j, \nu} \int_{\mathbf{R}^{d}} d z\left(2 E \left[\chi_{V^{c}(t, x+b, \varepsilon)}(z)\right.\right. \\
& \left.\left|\int_{0}^{t} d b_{\nu} \int_{\mathbf{R}^{d}} d v S_{j \nu}(z-v) f_{m, n}(x+b(s), v)(s)\right|^{2}\right] \\
& +\frac{1}{2} E\left[\chi_{V^{c}(t, x+b, \varepsilon)}(z)\right. \\
& \left.\left.\left|\int_{0}^{t} d s \int_{\mathbf{R}^{d}} d v S_{j \nu}(z-v) g_{m, n ; \nu}(x+b(s), v)\right|^{2}\right]\right)^{\eta / 2} \\
& =\sup _{t \in \mathcal{T}} d^{\eta / 2} c_{1} \sum_{j, \nu} \int_{\mathbf{R}^{d}} d z\left(2 E \left[\chi_{|x-z|>\varepsilon}(z)\right.\right. \\
& \left.\left|\int_{0}^{t \wedge \tau_{\varepsilon}(x-z)} d b_{\nu}(s) \int_{\mathbf{R}^{d}} d v S_{j \nu}(z-v) f_{m, n}(x+b(s), v)\right|^{2}\right] \\
& +\frac{1}{2} E\left[\chi_{|x-z|>\varepsilon}(z)\right. \\
& \left.\left.\left|\int_{0}^{t \wedge \tau_{\varepsilon}(x-z)} d s \int_{\mathbf{R}^{d}} d v S_{j \nu}(z-v) g_{m, n ; \nu}(x+b(s), v)\right|^{2}\right]\right)^{\eta / 2} \\
& =\sup _{t \in \mathcal{T}} d^{\eta / 2} c_{1} \sum_{j, \nu} \int_{\mathbf{R}^{d}} d z\left(2 E \left[\mid \int_{0}^{t \wedge \tau_{\varepsilon}(x-z)} d b_{\nu}(s)\right.\right. \\
& \left.\left.\int_{\mathbf{R}^{d}} d v \chi_{|x-z|>\varepsilon}(z) S_{j \nu}(z-v) f_{m, n}(x+b(s), v)\right|^{2}\right] \\
& +\frac{1}{2} E\left[\mid \int_{0}^{t \wedge \tau_{\varepsilon}(x-z)} d s\right. \\
& \left.\left.\left.\int_{\mathbf{R}^{d}} d v \chi_{|x-z|>\varepsilon}(z) S_{j \nu}(z-v) g_{m, n ; \nu}(x+b(s), v)\right|^{2}\right]\right)^{\eta / 2} \\
& =\sup _{t \in \mathcal{T}} d^{\eta / 2} c_{1} \sum_{j, \nu} \int_{\mathbf{R}^{d}} d z\left(2 E \left[\int_{0}^{t \wedge \tau_{\varepsilon}(x-z)} d s\right.\right. \\
& \left.\left|\int_{\mathbf{R}^{d}} d v \chi_{|x-z|>\varepsilon}(z) S_{j \nu}(z-v) f_{m, n}(x+b(s), v)\right|^{2}\right] \\
& +\frac{1}{2} E\left[\mid \int_{0}^{t \wedge \tau_{\varepsilon}(x-z)} d s\right. \\
& \left.\left.\left.\int_{\mathbf{R}^{d}} d v \chi_{|x-z|>\varepsilon}(z) S_{j \nu}(z-v) g_{m, n ; \nu}(x+b(s), v)\right|^{2}\right]\right)^{\eta / 2}
\end{aligned}
$$




$$
\begin{aligned}
& \leq d^{\eta / 2} c_{1} \sum_{j, \nu} \int_{\mathbf{R}^{d}} d z\left(2 E \left[\int_{0}^{t_{0}} d s\right.\right. \\
&\left.\left|\int_{\mathbf{R}^{d}} d v \chi_{V^{c}(s, x+b, \varepsilon)}(z) S_{j \nu}(z-v) f_{m, n}(x+b(s), v)\right|^{2}\right] \\
&+ \frac{1}{2} E\left[t_{0} \int_{0}^{t_{0}} d s \mid \int_{\mathbf{R}^{d}} d v \chi_{V^{c}(s, x+b, \varepsilon)}(z)\right. \\
&\left.\left.\left.S_{j \nu}(z-v) g_{m, n ; \nu}(x+b(s), v)\right|^{2}\right]\right)^{\eta / 2}
\end{aligned}
$$

Since $\operatorname{supp} \rho_{1 / n}, \operatorname{supp} \rho_{1 / m} \subset\left\{z \in \mathbf{R}^{d}|| z \mid \leq \frac{\varepsilon}{2}\right\}$,

$$
\begin{aligned}
& \left|\int_{\mathbf{R}^{d}} d v \chi_{V^{c}(s, x+b, \varepsilon)}(z) S_{j \nu}(z-v) f_{m, n}(x+b(s), v)\right| \\
& \quad \leq c_{2} \sup _{|x+b(s)-v| \leq \varepsilon / 2}\left(\chi_{V^{c}(s, x+b, \varepsilon)}(z) \frac{1}{|z-v|^{d-1}}\right) \\
& \quad \leq H(x+b(s), z)
\end{aligned}
$$

and

$$
\begin{aligned}
& \left|\int_{\mathbf{R}^{d}} d v \chi_{V^{c}(s, x+b, \varepsilon)}(z) S_{j \nu}(z-v) g_{m, n ; \nu}(x+b(s), v)\right| \\
& \quad \leq c_{2} \sup _{|x+b(s)-v| \leq \varepsilon / 2}\left(\chi_{V^{c}(s, x+b, \varepsilon)}(z) \frac{1}{|z-v|^{d-1}}\right) \\
& \quad+c_{3} \sup _{|x+b(s)-v| \leq \varepsilon / 2}\left(\chi_{V^{c}(s, x+b, \varepsilon)}(z) \partial_{\nu} \frac{1}{|z-v|^{d-1}}\right) \\
& \quad \leq H(x+b(s), z)
\end{aligned}
$$

hold, where

$$
H(x+y, z)= \begin{cases}C_{\varepsilon}, & |x+y| \geq \frac{|z|}{4} \\ C_{\varepsilon}\left(1 \wedge \frac{1}{|z|^{d-1}}\right), & |x+y|<\frac{|z|}{4}\end{cases}
$$

with constants $c_{2}, c_{3}$ and $C_{\varepsilon}$. Also, by the definition of $f_{m, n}$ and $g_{m, n ; \nu}$,

$$
\begin{gathered}
\left|\int_{\mathbf{R}^{d}} d v \chi_{V^{c}(s, x+b, \varepsilon)}(z) S_{j \nu}(z-v) f_{m, n}(x+b(s), v)\right| \rightarrow 0 \\
\left|\int_{\mathbf{R}^{d}} d v \chi_{V^{c}(s, x+b, \varepsilon)}(z) S_{j \nu}(z-v) g_{m, n ; \nu}(x+b(s), v)\right| \rightarrow 0 \\
\text { as } m, n \rightarrow \infty
\end{gathered}
$$


hold. Thus if we show

$$
\int_{\mathbf{R}^{d}} E\left[\int_{0}^{t_{0}} H^{2}(x+b(s), z) d s\right]^{\eta / 2} d z<\infty
$$

then we have by Lebesgue's dominated convergence theorem that the second term of (4.8) converges to 0 as $n, m \rightarrow \infty$, because the left hand sides of (4.11) and (4.12) tend to 0 as $m, n \rightarrow \infty$. Finally we shall show (4.13). Since

$$
P\left[\sup _{s \in \mathcal{T}}|x+b(s)| \geq \frac{|z|}{4}\right] \leq 2 d \exp \left(-\frac{1}{2 d t_{0}}(|z| / 4-|x|)_{+}^{2}\right)
$$

we have

$$
\begin{gathered}
E\left[H^{2}(x+b(s), z)\right]=E\left[H^{2}(x+b(s), z) ; \sup _{s \in \mathcal{T}}|x+b(s)| \geq \frac{|z|}{4}\right] \\
+E\left[H^{2}(x+b(s), z) ; \sup _{s \in \mathcal{T}}|x+b(s)|<\frac{|z|}{4}\right] \\
\leq C_{\varepsilon}^{2}\left(P\left[\sup _{s \in \mathcal{T}}|x+b(s)| \geq \frac{|z|}{4}\right]+\left(1 \wedge \frac{1}{|z|^{2(d-1)}}\right)\right),
\end{gathered}
$$

the right hand side in (4.14) is integrable.

Lemma 4.4 For each $t \in \mathcal{T}, f \in \mathcal{D}\left(\mathbf{R}^{d}\right),\left\{T_{t}^{A, n} f\right\}_{n=1}^{\infty}$ is a Cauchy sequence in $L^{2}\left(\mathcal{S}^{\prime}\left(\mathbf{R}^{d} ; \mathbf{R}^{d}\right) \times \mathbf{R}^{d}, \mu \times d x\right)$, where $d x$ denotes the Lebesgue measure in $\mathbf{R}^{d}$. Moreover, this convergence is uniform in $t \in \mathcal{T}$,

$$
\sup _{t \in \mathcal{T}}\left\|T_{t}^{A, n} f-T_{t}^{A, m} f\right\|_{L^{2}\left(\mathcal{S}^{\prime}\left(\mathbf{R}^{d} ; \mathbf{R}^{d}\right) \times \mathbf{R}^{d}\right)} \rightarrow 0 \quad \text { as } \quad m, n \rightarrow \infty .
$$

Proof. Since $T_{t}^{A, n} f(x)$ is continuous with respect to $x \in \mathbf{R}^{d}, T_{t}^{A, n} f$ is $\mathcal{B}\left(\mathcal{S}^{\prime}\left(\mathbf{R}^{d} ; \mathbf{R}^{d}\right) \times \mathbf{R}^{d}\right)$ measurable.

By (4.6) and Fubini's theorem,

$$
\begin{aligned}
\left\|T_{t}^{A, n} f-T_{t}^{A, m} f\right\|_{L^{2}\left(\mathcal{S}^{\prime}\left(\mathbf{R}^{d} ; \mathbf{R}^{d}\right) \times \mathbf{R}^{d}\right)}^{2} & \\
\quad & \int_{\mathbf{R}^{d}} F_{m, n}^{t}(x) d x \\
& \leq 2 \int_{\mathbf{R}^{d}} E\left[|f(x+b(t))|^{2}\left\{1-\exp \left(-\int_{\mathbf{R}^{d}} \psi_{m, n}(t, z, x) d z\right)\right\}\right] d x \\
& =2 \int_{B_{k}(0)} E\left[|f(x+b(t))|^{2}\left\{1-\exp \left(-\int_{\mathbf{R}^{d}} \psi_{m, n}(t, z, x) d z\right)\right\}\right] d x
\end{aligned}
$$




$$
+2 \int_{B_{k}(0)^{c}} E\left[|f(x+b(t))|^{2}\left\{1-\exp \left(-\int_{\mathbf{R}^{d}} \psi_{m, n}(t, z, x) d z\right)\right\}\right] d x
$$

where $B_{k}(0)$ is a closed ball with radius $k$ and center 0 . The second term of (4.15) is dominated as follows:

$$
\begin{gathered}
\int_{B_{k}(0)^{c}} E\left[|f(x+b(t))|^{2}\left\{1-\exp \left(-\int_{\mathbf{R}^{d}} \psi_{m, n}(t, z, x) d z\right)\right\}\right] d x \\
=\int_{B_{k}(0)^{c}} E\left[1_{\left\{\inf _{0 \leq s \leq t}|x+b(s)| \leq r\right\}}|f(x+b(t))|^{2}\right. \\
\left.\left\{1-\exp \left(-\int_{\mathbf{R}^{d}} \psi_{m, n}(t, z, x) d z\right)\right\}\right] d x \\
\leq M^{2} \int_{B_{k}(0)^{c}} P\left[\left\{\omega\left|\inf _{0 \leq s \leq t_{0}}\right| x+b(s) \mid \leq r\right\}\right] d x
\end{gathered}
$$

where $r>0$ and $M>0$ are constants such that

$$
\operatorname{supp} f \subset\{|x| \leq r\}, \quad \max _{\mathbf{R}^{d}}|f| \leq M .
$$

Since $P\left[\left\{\omega\left|\inf _{0 \leq s \leq t_{0}}\right| x+b(s) \mid \leq r\right\}\right]$ is integrable with respect to $d x$ in $\mathbf{R}^{d}$, the second term of (4.15) converges to 0 uniformly in $t \in \mathcal{T}$ and $m, n$ as $k \rightarrow \infty$.

Next, the first term of (4.15) is dominated as follows: For any $k>0$,

$$
\begin{aligned}
& \sup _{t \in \mathcal{T}} \int_{B_{k}(0)} E\left[|f(x+b(t))|^{2}\left(1-\exp \left(-\int_{\mathbf{R}^{d}} \psi_{m, n}(t, z, x) d z\right)\right)\right] d x \\
& \leq M^{2} \int_{B_{k}(0)} \sup _{t \in \mathcal{T}} E\left[1-\exp \left(-\int_{\mathbf{R}^{d}} \psi_{m, n}(t, z, x) d z\right)\right] d x
\end{aligned}
$$

By Lemma 4.3, the integrand in the right hand side of (4.17) tends to 0 as $n, m \rightarrow \infty$. Since $B_{k}(0)$ is compact, we have by the bounded convergence theorem that (4.17) converges to 0 as $n, m \rightarrow \infty$.

\section{Construction of the Generator}

First of all, we introduce a notion of a semi-group process. Let $(\Omega, \mathcal{F}, P)$ be a probability space. Let $X$ be a Hilbert space. We denote the space of all continuous endomorphism of $X$ by $\mathcal{L}(X)$.

Definition 5.1 If an $\mathcal{L}(X)$-valued continuous process $\left\{T_{t}\right\}_{t \geq 0}$ satisfies 
that

$$
\begin{aligned}
P\left(\left\{T_{t}\right\}_{t \geq 0}\right. & \text { is a (strongly continuous) } \\
& \text { symmetric contraction semi-group) }=1,
\end{aligned}
$$

we call it a (strongly continuous) semi-group process on $X$ with respect to $P$.

Remark 5.2. In this case, by contractivity and symmetricity, $\left\{T_{t}\right\}_{t \geq 0}$ is a self-adjoint semi-group $P$-a.s.

In this section, we shall construct a semi-group process on $L^{2}\left(\mathbf{R}^{d}\right)$ whose generator is formally expressed as " $(\partial-i A)^{2}$ " and show it is self-adjoint. First, let us consider the problem in a general setting.

Theorem 5.3 Let $\left\{T_{t}^{n}\right\}_{t \geq 0}(n=1,2, \cdots)$ be strongly continuous semigroup processes. If there exists a countable dense subset $\mathcal{D}$ of $X$ such that for any $f \in \mathcal{D}, \varepsilon>0$ and $T>0$,

$$
\lim _{m, n \rightarrow \infty} \sup _{t \in[0, T]} P\left(\left\|T_{t}^{n} f-T_{t}^{m} f\right\|_{X}>\varepsilon\right)=0 .
$$

Then there exists a strongly continuous semi-group process $\left\{T_{t}\right\}_{t \geq 0}$ such that

$$
\lim _{n \rightarrow \infty} \sup _{t \in[0, T]} P\left(\left\|T_{t}^{n} f-T_{t} f\right\|_{X}>\varepsilon\right)=0 \quad \text { for } f \in X .
$$

Proof. Without loss of generality, we may assume that for any $\omega \in \Omega$, $\left\{T_{t}^{n}(\omega)\right\}_{t \geq 0}$ is a strongly continuous symmetric contraction semi-group for any $n \in \mathbf{N}$. By (5.1) and diagonal method, we can select a subsequence $\left\{n_{k}\right\}_{k=1}^{\infty}$ such that, for each $f \in \mathcal{D}$,

$$
\sum_{l=1}^{\infty} \frac{1}{2^{l}} \sup _{0 \leq t \leq l} P\left(\left\|T_{t}^{n_{k}} f-T_{t}^{n_{k+1}} f\right\|_{X}>\frac{1}{2^{k}}\right)<\frac{1}{2^{k}},
$$

except for a finite number of $k$ 's which may depend on $f$. We set,

$$
\Lambda_{k, t, f} \equiv\left\{\left\|T_{t}^{n_{k}} f-T_{t}^{n_{k+1}} f\right\|_{X}>\frac{1}{2^{k}}\right\} .
$$

By using Borel-Cantelli's lemma, $P\left(\liminf _{k \rightarrow \infty} \Lambda_{k, t, f}^{c}\right)=1$, for $f \in \mathcal{D}$. For any $\omega \in \liminf _{k \rightarrow \infty} \Lambda_{k, t, f}^{c},\left\{T_{t}^{n_{k}} f\right\}_{k=1}^{\infty}$ is a Cauchy sequence in $X$. Since $\mathcal{D}$ is contained in $X$ densely and $\operatorname{Card}(\mathcal{D})=\aleph_{0}$, for any $f \in X,\left\{T_{t}^{n_{k}} f\right\}_{k=1}^{\infty}$ is a Cauchy sequence in $X$. We denote the limit of $\left\{T_{t}^{n_{k}} f\right\}_{k=1}^{\infty}$ by $T_{t} f$. It is 
obvious that $T_{t}$ is a linear operator and $\left\|T_{t}\right\| \leq 1$. Then we have

$$
P\left(T_{t}^{n_{k}} f \rightarrow T_{t} f \quad \text { in } \quad X, \quad \text { for } f \in X\right)=1 .
$$

In the following, we shall construct semi-group process $\left\{T_{t}\right\}_{t \geq 0}$.

Step 1 (strong continuity)

Let $\left\{t_{n}\right\}_{n=1}^{\infty} \subset \mathbf{Q} \cap[0,1]$ be a decreasing sequence which converges to 0 . Then

$$
P\left(\lim _{n \rightarrow \infty}\left\|T_{t_{n}} f-f\right\|_{X}=0, \quad \text { for } f \in X\right)=1
$$

holds.

Proof. Because $\left\{T_{t}^{n}\right\}_{t \geq 0}$ are contraction semi-groups and (5.3), we can show that $\left\|T_{t} f\right\|_{X} \leq\|f\|_{X} P$-a.s for any $t \geq 0$. For any $k \in \mathbf{N}, f \in \mathcal{D}$, $\left\|T_{t}^{n_{k}} f-f\right\|_{X}$ is an increasing function with respect to $t \geq 0$. In fact, by the spectrum representation of the self-adjoint operator,

$$
\left\|T_{t}^{n_{k}} f-f\right\|_{X}^{2}=\int_{0}^{\infty}\left(1-e^{-t \lambda}\right)^{2} d\left\|E_{\lambda}^{n_{k}} f\right\|^{2} .
$$

$\left(1-e^{-t \lambda}\right)^{2}$ is an increasing function of $t$, then we can show this.

Since $\left\|T_{t} f-f\right\|_{X}$ is the limit of the sequence of the increasing functions $\left\|T_{t}^{n_{k}} f-f\right\|_{X},\left\|T_{t} f-f\right\|_{X}$ is also increasing. For any $\varepsilon>0$ and $m \in \mathbf{N}$,

$$
\begin{aligned}
& P\left(\lim _{n \rightarrow \infty}\left\|T_{t_{n}} f-f\right\|_{X}>\varepsilon\right) \leq P\left(\left\|T_{t_{m}} f-f\right\|_{X}>\varepsilon\right) \\
& \quad \leq P\left(\left\|T_{t_{m}}^{n_{k}} f-T_{t_{m}} f\right\|_{X}>\varepsilon / 2\right)+P\left(\left\|T_{t_{m}}^{n_{k}} f-f\right\|_{X}>\varepsilon / 2\right) .
\end{aligned}
$$

By the definition of $T_{t} f$, for any $T>1$,

$$
\begin{aligned}
\sup _{t \in \mathbf{Q} \cap[0, T]} P\left(\left\|T_{t}^{n} f-T_{t} f\right\|_{X}>\varepsilon\right) \\
=\sup _{t \in \mathbf{Q} \cap[0, T]} P\left(\lim _{k \rightarrow \infty}\left\|T_{t}^{n} f-T_{t}^{n_{k}} f\right\|_{X}>\varepsilon\right) \\
\quad \leq \sup _{t \in \mathbf{Q} \cap[0, T]} \lim _{k \rightarrow \infty} \inf _{k \rightarrow \infty} P\left(\left\|T_{t}^{n} f-T_{t}^{n_{k}} f\right\|_{X}>\varepsilon\right) \\
\quad \leq \lim _{k \rightarrow \infty} \inf _{t \in \mathbf{Q} \cap[0, T]} P\left(\left\|T_{t}^{n} f-T_{t}^{n_{k}} f\right\|_{X}>\varepsilon\right) .
\end{aligned}
$$

Then, the first term of (5.6) can be arbitrarily small independently of $t \in$ $\mathbf{Q} \cap[0, T]$, the second term tends to 0 as $m \rightarrow \infty$. Since $\mathcal{D}$ is contained in $X$ densely and Card $(\mathcal{D})=\aleph_{0}$, we have (5.4). 
Now we set

$$
\begin{array}{ll}
\Omega_{t} \equiv\left\{\omega \in \Omega: T_{t}^{n_{k}} f \rightarrow T_{t} f \quad \text { in } \quad X,\right. & \text { for } f \in X\} \\
\Omega_{0} \equiv\left\{\omega \in \Omega: \lim _{n \rightarrow \infty}\left\|T_{t_{n}} f-f\right\|_{X}=0,\right. & \text { for } f \in X\}
\end{array}
$$

and

$$
\Omega_{1} \equiv \cap_{t \in \mathbf{Q} \cap[0, \infty)} \Omega_{t} \bigcap \Omega_{0}
$$

We readily see $P\left(\Omega_{1}\right)=1$.

Remark 5.4. $\Omega_{1}$ does not depend on the decreasing sequence $\left\{t_{n}\right\}_{n=1}^{\infty}$, because $\left\|T_{t} f-f\right\|_{X}$ is increasing with respect to $t \geq 0$.

Step 2 (semi-group property)

For any $\omega \in \Omega_{1}, t \in \mathbf{Q} \cap[0, \infty)$,

$$
T_{s} \circ T_{t}=T_{t} \circ T_{s}=T_{t+s}
$$

Proof. For any $f \in X$,

$$
\begin{aligned}
& \left\|T_{s} \circ T_{t} f-T_{s+t} f\right\|_{X} \\
& \quad \leq\left\|T_{s} \circ T_{t} f-T_{s}^{n_{k}} \circ T_{t} f\right\|_{X}+\left\|T_{s}^{n_{k}} \circ T_{t} f-T_{s}^{n_{k}} \circ T_{t}^{n_{k}} f\right\|_{X} \\
& \quad+\left\|T_{s}^{n_{k}} \circ T_{t}^{n_{k}} f-T_{t+s}^{n_{k}} f\right\|_{X}+\left\|T_{t+s}^{n_{k}} f-T_{t+s} f\right\|_{X} .
\end{aligned}
$$

The third term of the right-hand side of (5.12) is 0 , because of the semigroup property of $\left\{T_{t}^{n_{k}}\right\}_{t \geq 0}$. By (5.8), the first and fourth terms tend to 0 as $k \rightarrow \infty$. Finally, for the second term, we have

$$
\begin{aligned}
\left\|T_{s}^{n_{k}} \circ T_{t} f-T_{s}^{n_{k}} \circ T_{t}^{n_{k}} f\right\|_{X} & \leq\left\|T_{s}^{n_{k}}\right\| \cdot\left\|T_{t} f-T_{t}^{n_{k}} f\right\|_{X} \\
& \leq\left\|T_{t} f-T_{t}^{n_{k}} f\right\|_{X} .
\end{aligned}
$$

Then we get (5.11).

\section{Step 3 (construction)}

For any $s \in[0, \infty)$, we take $\left\{s_{n}\right\} \subset \mathbf{Q} \cap[0, \infty)$ such that $s_{n} \downarrow s$. By the semi-group property, contraction property and Step 1, we can show that for any $f \in X,\left\{T_{s_{n}} f\right\}_{n=1}^{\infty}$ is a Cauchy sequence in $X$. Therefore we define the operator $\left\{T_{t}\right\}_{t \geq 0}$ as follows.

For $\omega \in \Omega_{1}$ and $f \in X$,

$$
T_{t} f \equiv \lim _{n \rightarrow \infty} T_{t_{n}} f .
$$


Then for any $\omega \in \Omega_{1},\left\{T_{t}\right\}_{t \geq 0}$ is a strongly continuous symmetric contraction semi-group. In fact, for any $\omega \in \Omega_{1}$, by Step 1 and the definition of $\left\{T_{t}\right\}_{t \geq 0}$,

$$
T_{t} f \rightarrow f \quad \text { as } t \rightarrow 0 \quad \text { in } X .
$$

Hence the semi-group $\left\{T_{t}\right\}_{t \geq 0}$ is strongly continuous. By the definition of $\left\{T_{t}\right\}_{t \geq 0}$, it is contractive. Because $\left\{T_{t}^{n_{k}}\right\}_{t \geq 0}$ is symmetric and $T_{t}^{n_{k}} \rightarrow$ $T_{t}$ strongly, it is obvious that $\left\{T_{t}\right\}_{t \geq 0}$ is a symmetric semi-group. Since $\left\{T_{t}^{n_{k}}\right\}_{t \geq 0}$ and $\left\{T_{t}\right\}_{t \geq 0}$ are strongly continuous, we have (5.2) by (5.7) easily. Then we can conclude this theorem.

Remark 5.5. By (5.14), we may denote

$$
\Omega_{0}=\left\{\omega \in \Omega: \lim _{t \rightarrow 0}\left\|T_{t} f-f\right\|_{X}=0, \quad \text { for } f \in X\right\} .
$$

In our case, $\left\{T_{t}^{A, n}\right\}_{t \geq 0}(n=1,2, \cdots) A \in \mathcal{S}^{\prime}\left(\mathbf{R}^{d} ; \mathbf{R}^{d}\right)$ are strongly continuous semi-group processes on $L^{2}\left(\mathbf{R}^{d}\right)$. By Lemma 4.4, we can easily check they satisfy the condition of Theorem 5.3. Therefore there is a strongly continuous symmetric contraction semi-group $\left\{T_{t}^{A}\right\}_{t \geq 0}$ for $\mu$-almost surely. Hence there exists a positive self-adjoint operator $\mathcal{G}_{A}$ for $\mu$-almost surely which generates the semi-group $\left\{T_{t}^{A}\right\}_{t \geq 0}$.

\section{Gauge Covariance}

Our purpose in this section is to show the gauge covariance of the generator $\mathcal{G}_{A}$. For any $\lambda \in L_{l o c}^{1}\left(\mathbf{R}^{d}\right)$, we set

$$
\lambda^{n}(x) \equiv w_{n}(x)\left\langle\rho_{1 / n}(x-\cdot), \lambda\right\rangle .
$$

Lemma 6.1 Let $\lambda \in L_{\text {loc }}^{1}\left(\mathbf{R}^{d}\right)$. For any $f \in L^{2}\left(\mathbf{R}^{d}\right)$, we have

$$
\begin{aligned}
& e^{i \lambda^{n}} f \rightarrow e^{i \lambda} f \quad \text { in } \quad L^{2}\left(\mathbf{R}^{d}\right) \\
& e^{-i \lambda^{n}} f \rightarrow e^{-i \lambda} f \quad \text { in } \quad L^{2}\left(\mathbf{R}^{d}\right) .
\end{aligned}
$$

Proof. We only show here (6.1). For any $f \in \mathcal{D}\left(\mathbf{R}^{d}\right)$, we have

$$
\begin{aligned}
\left\|e^{i \lambda^{n}} f-e^{i \lambda} f\right\|_{L^{2}\left(\mathbf{R}^{d}\right)}^{2} & =\left\|f-e^{i\left(\lambda-\lambda^{n}\right)} f\right\|_{L^{2}\left(\mathbf{R}^{d}\right)}^{2} \\
& =' 2 \int_{\mathbf{R}^{d}}|f|^{2}\left|1-\cos \left(\lambda-\lambda^{n}\right)\right| d x
\end{aligned}
$$




$$
\leq 2 M^{2} \int_{|x| \leq r}\left|1-\cos \left(\lambda-\lambda^{n}\right)\right| d x
$$

where $M, r$ are constants such that $M=\sup |f|, \operatorname{supp} f \subset\{|x| \leq r\}$. Because of the inequality $|1-\cos x| \leq|x|$, we have

$$
\begin{aligned}
(6.3) \leq & 2 M^{2} \int_{|x| \leq r}\left|\lambda-\lambda^{n}\right| d x \\
\leq & 2 M^{2} \int_{|x| \leq r}\left|1-w_{n}(x)\right| \cdot|\lambda| d x \\
& +\left\|w_{n}\right\|_{\infty} \int_{|x| \leq r}\left|\lambda-\left\langle\rho_{1 / n}, \lambda\right\rangle\right| d x .
\end{aligned}
$$

The last term of (6.4) tends to 0 as $n \rightarrow \infty$, because of the definition of $w_{n}$ and $\lambda \in L_{l o c}^{1}\left(\mathbf{R}^{d}\right)$.

Since $e^{i \lambda^{n}}$ and $e^{i \lambda}$ are contraction operators on $L^{2}\left(\mathbf{R}^{d}\right)$ and $\mathcal{D}\left(\mathbf{R}^{d}\right)$ is dense in $L^{2}\left(\mathbf{R}^{d}\right), e^{i \lambda^{n}} f$ is $L^{2}$ convergent to $e^{i \lambda} f$ for any $f \in L^{2}\left(\mathbf{R}^{d}\right)$.

Theorem 6.2 Let $\lambda \in L_{\text {loc }}^{1}\left(\mathbf{R}^{d}\right)$. We have

$$
\begin{gathered}
\mu\left(e^{i \lambda^{n}} T_{t}^{A, n} e^{-i \lambda^{n}} f \rightarrow e^{i \lambda} T_{t}^{A} e^{-i \lambda} f \quad \text { in } \quad L^{2}\left(\mathbf{R}^{d}\right)\right. \\
\text { for } \left.f \in L^{2}\left(\mathbf{R}^{d}\right)\right)=1,
\end{gathered}
$$

where $e^{i \lambda^{n}}, e^{-i \lambda^{n}}$ and $e^{i \lambda}$ are multiplication operators on $L^{2}\left(\mathbf{R}^{d}\right)$.

Proof. Let $\Omega_{1}$ be the subset of $\mathcal{S}^{\prime}\left(\mathbf{R}^{d} ; \mathbf{R}^{d}\right)$ which was defined in (5.10) and let $\left\{n_{k}\right\}_{k=1}^{\infty}$ be an associated subsequence with $\Omega_{1}$. For any $A \in \Omega_{1}$ and $f \in L^{2}\left(\mathbf{R}^{d}\right)$,

$$
\begin{aligned}
& \left\|e^{i \lambda} T_{t}^{A} e^{-i \lambda} f-e^{i \lambda^{n_{k}}} T_{t}^{A, n_{k}} e^{-i \lambda^{n_{k}}} f\right\|_{L^{2}\left(\mathbf{R}^{d}\right)} \\
& \leq\left\|e^{i \lambda} T_{t}^{A} e^{-i \lambda} f-e^{i \lambda_{k}} T_{t}^{A} e^{-i \lambda} f\right\|_{L^{2}\left(\mathbf{R}^{d}\right)} \\
& \quad+\left\|e^{i \lambda_{k}} T_{t}^{A} e^{-i \lambda} f-e^{i \lambda^{n_{k}}} T_{t}^{A, n_{k}} e^{-i \lambda} f\right\|_{L^{2}\left(\mathbf{R}^{d}\right)} \\
& \quad+\left\|e^{i \lambda^{n_{k}}} T_{t}^{A, n_{k}} e^{-i \lambda} f-e^{i \lambda^{n_{k}}} T_{t}^{A, n_{k}} e^{-i \lambda^{n_{k}}} f\right\|_{L^{2}\left(\mathbf{R}^{d}\right)} \\
& \leq\left\|e^{i \lambda} T_{t}^{A} e^{-i \lambda} f-e^{i \lambda^{n_{k}}} T_{t}^{A} e^{-i \lambda} f\right\|_{L^{2}\left(\mathbf{R}^{d}\right)} \\
& \quad+\left\|T_{t}^{A} e^{-i \lambda} f-T_{t}^{A, n_{k}} e^{-i \lambda} f\right\|_{L^{2}\left(\mathbf{R}^{d}\right)} \\
& \quad+\left\|e^{-i \lambda} f-e^{-i \lambda^{n}} f\right\|_{L^{2}\left(\mathbf{R}^{d}\right) .}
\end{aligned}
$$

By using Lemma 6.1, the first and third terms in (6.6) tend to 0 as $k \rightarrow \infty$. By $(5,8)$ the second term in $(6.6)$ also tends to 0 as $k \rightarrow \infty$. 
We shall define

$$
(A+\partial \lambda)^{n}(x) \equiv A^{n}(x)+w_{n}(x)\left\langle\rho_{1 / n}(x-\cdot), \partial \lambda\right\rangle .
$$

Then we have, by the definition of the semi-groups $\left\{T_{t}^{A, n}\right\}_{t \geq 0}$ and $\left\{T_{t}^{(A+\partial \lambda), n}\right\}_{t \geq 0}$, for any $A \in \mathcal{S}^{\prime}\left(\mathbf{R}^{d} ; \mathbf{R}^{d}\right), f \in L^{2}\left(\mathbf{R}^{d}\right)$ and $T>0$,

$$
\left\|e^{i \lambda^{n}} T_{t}^{A, n} e^{-i \lambda^{n}} f-T_{t}^{(A+\partial \lambda), n} f\right\|_{L^{2}\left(\mathbf{R}^{d}\right)} \rightarrow 0 \quad \text { as } n \rightarrow \infty .
$$

Moreover this convergence is uniform in $t \in[0, T]$. Since $\left\{T_{t}^{A, n}\right\}_{t \geq 0}$ and $\left\{T_{t}^{A}\right\}_{t \geq 0}$ are strongly continuous semi-group processes, we have for any $T>$ 0 , the convergence $[6.6)$ is also uniform in $t \in[0, T]$. Then we can obtain, for any $f \in \mathcal{D}\left(\mathbf{R}^{d}\right), \varepsilon>0$ and $T>0$,

$$
\lim _{m, n \rightarrow \infty} \sup _{t \in[0, T]} \mu\left(\left\|T_{t}^{A+\partial \lambda, n} f-T_{t}^{A+\partial \lambda, m} f\right\|_{L^{2}\left(\mathbf{R}^{d}\right)}>\varepsilon\right)=0 .
$$

By Theorem 5.3, we have a strongly continuous symmetric semi-group process $\left\{T_{t}^{A+\partial \lambda}\right\}_{t \geq 0}$ as the limit of $\left\{T_{t}^{A+\partial \lambda, n}\right\}_{t \geq 0}$. We denote its generator by $\mathcal{G}_{A+\partial \lambda}$. (6.7) implies that the semi-group process $\left\{T_{t}^{A+\partial \lambda}\right\}_{t \geq 0}$ coincides with the semi-group process $\left\{e^{i \lambda} T_{t}^{A} e^{-i \lambda}\right\}_{t \geq 0}$. Thus, the following theorem holds.

Theorem 6.3 Let $\lambda \in L_{\text {loc }}^{1}\left(\mathbf{R}^{d}\right)$. We have

$$
\mu\left(e^{i \lambda} \mathcal{G}_{A} e^{-i \lambda}=\mathcal{G}_{A+\partial \lambda}\right)=1 .
$$

\section{The Spectra of the Generator}

This section deals with the study of spectra of $\mathcal{G}_{A}, A \in \mathcal{S}^{\prime}\left(\mathbf{R}^{d} ; \mathbf{R}^{d}\right)$. Let $\left\{\theta_{z}\right\}_{z \in \mathbf{R}^{d}}$ be an ergodic family of measure preserving transformations defined in section 3. Let $\left\{\tau_{z}\right\}_{z \in \mathbf{R}^{d}}$ be an unitary operator

$$
\tau_{z} f=f(\cdot-z) \quad f \in L^{2}\left(\mathbf{R}^{d}\right) .
$$

We define $\left\{T_{t}^{\theta_{z} A}\right\}_{t>0}$ as follows. For any $f \in L^{2}\left(\mathbf{R}^{d}\right)$,

$$
T_{t}^{\theta_{z} A} f \equiv \lim _{k \rightarrow \infty} T_{t}^{\theta_{z} A, n_{k}} f \quad \text { in } \quad L^{2}\left(\mathbf{R}^{d}\right)
$$

Lemma 7.1 For any $z \in \mathbf{R}^{d}$, there exists a semi-group process $\left\{T_{t}^{\theta_{z} A}\right\}_{t \geq 0}$ $\mu$-almost surely. Moreover for any $t>0$, we have

$$
T_{t}^{\theta_{z} A}=\tau_{z}^{*} T_{t}^{A} \tau_{z}
$$


where $\tau_{z}^{*}$ is an adjoint operator of $\tau_{z}$.

Proof. For any $f \in L^{2}\left(\mathbf{R}^{d}\right)$ and $t \in \mathbf{Q} \cap[0, \infty)$,

$$
\begin{aligned}
\left\|T_{t}^{\theta_{z} A, n_{k}} f-T_{t}^{\theta_{z} A, n_{l}} f\right\|_{L^{2}\left(\mathbf{R}^{d}\right)} & =\left\|\tau_{z}^{*} T_{t}^{A, n_{k}} \tau_{z} f-\tau_{z}^{*} T_{t}^{A, n_{l}} \tau_{z} f\right\|_{L^{2}\left(\mathbf{R}^{d}\right)} \\
& =\left\|T_{t}^{A, n_{k}} \tau_{z} f-T_{t}^{A, n_{l}} \tau_{z} f\right\|_{L^{2}\left(\mathbf{R}^{d}\right)} .
\end{aligned}
$$

By (5.3), the right hand side of (7.2) converges to 0 as $k, l \rightarrow \infty$. Then we get $\left\{T_{t}^{\theta_{z} A, n_{k}} f\right\}_{k=1}^{\infty}$ is a Cauchy sequence in $L^{2}\left(\mathbf{R}^{d}\right) \mu$-almost surely, because $\left\{T_{t}^{A, n_{k}} f\right\}_{k=1}^{\infty}$ is a Cauchy sequence. And we have

$$
\begin{aligned}
\left\|T_{t}^{\theta_{z} A} f-\tau_{z}^{*} T_{t}^{A} \tau_{z} f\right\|_{L^{2}\left(\mathbf{R}^{d}\right)} & \leq\left\|T_{t}^{\theta_{z} A} f-T_{t}^{\theta_{z} A, n_{k}} f\right\|_{L^{2}\left(\mathbf{R}^{d}\right)} \\
& +\left\|T_{t}^{\theta_{z} A, n_{k}} f-\tau_{z}^{*} T_{t}^{A, n_{k}} \tau_{z} f\right\|_{L^{2}\left(\mathbf{R}^{d}\right)} \\
& +\left\|\tau_{z}^{*} T_{t}^{A, n_{k}} \tau_{z} f-\tau_{z}^{*} T_{t}^{A} \tau_{z} f\right\|_{L^{2}\left(\mathbf{R}^{d}\right)} .
\end{aligned}
$$

The second term is 0 . The first and third terms tend to 0 as $k$ to infinity. For any $t \geq 0, T_{t}^{\theta_{z} A}$ was defined by (5.13), then we obtain (7.1).

Let $\sigma(A), \sigma_{p p}(A), \sigma_{a c}(A)$ and $\sigma_{s c}(A)$ be the spectrum, point spectrum, absolutely continuous spectrum and singular continuous spectrum of $\mathcal{G}_{A}$ respectively.

Theorem 7.2 There exist closed subsets $\sigma, \sigma_{p p}, \sigma_{a c}$ and $\sigma_{s c}$ of $\mathbf{R}$ such that $\sigma=\sigma(A), \sigma_{p p}=\sigma_{p p}(A), \sigma_{a c}=\sigma_{a c}(A)$ and $\sigma_{s c}=\sigma_{s c}(A)$ for almost $\mu$-surely.

Proof. Let $E(\Lambda, A), \Lambda \in \mathcal{B}\left(\mathbf{R}^{d}\right)$, be the resolution of the identity of the operator $\mathcal{G}_{A}$ and let

$$
E(\cdot, A)=E_{p p}(\cdot, A)+E_{a c}(\cdot, A)+E_{s c}(\cdot, A)
$$

be its Lebesgue decomposition. By Lemma 7.1, we have

$$
E_{\#}(\cdot, A)=\tau_{z}^{*} E_{\#}(\cdot, A) \tau_{z},
$$

where \# $=p p, a c$ and $s c$. Then there exists a closed subset $\sigma_{\#}$ of $\mathbf{R}$ such that topological support of $E_{\#}(\cdot, A)$ is $\sigma_{\#}$ for $\mu$-almost surely. (cf. [C] Proposition V.2.4).

Acknowledgments The author would like to thank Professor S. Albeverio, Professor T. Ichinose, Professor S. Nakao, Professor S. Takanobu and 
Professor H. Tamura for many significant suggestions. He is also grateful to the referee for a lot of comments and encouragement.

\section{References}

[AHK 1] Albeverio S. and Høegh-Krohn R., Euclidean Markov fields and relativistic quantum fields from stochastic partial differential equations in four dimensions. Phys. Lett. B 177 (1986), 175-179.

[AHK 2] Albeverio S. and Høegh-Krohn R., Construction of interacting local relativistic quantum fields in four space-time dimensions. Phys. Lett. B 200 (1988), 108-114; Err. B 202 (1988), 621.

[AHKI] Albeverio S., Høegh-Krohn R. and Iwata K., Covariant Markovian random fields in four space-time dimensions with nonlinear electromagnetic interaction. in Applications of self-adjoint extensions in quantum physics, Lect. Notes in Phys. 324. Springer-Verlag, Berlin, 1989.

[AHKHK] Albeverio S., Høegh-Krohn R., Holden H. and Kolsrud T., Construction of quantized Higgs-like fields in two dimensions. Phys. Lett. B 222 (1989), 263-268.

[AIK 1] Albeverio S., Iwata K. and Kolsrud T., Random fields as solutions of the inhomogeneous quaternionic Cauchy-Riemann equation I. Invariance and analytic continuation. Comm. Math. Phys. 132 (1990), 555-580.

[AIK 2] Albeverio S., Iwata K. and Kolsrud T., A model of four space-time dimensional gauge fields: reflection positivity for associated random currents. 257-269 in Rigorous results in quantum dynamics, Ed. J. Dittrich, P. Exner (World Scientific, Singapore, 1991).

[AIK 3] Albeverio S., Iwata K. and Kolsrud T., Homogeneous Markov generalized vector fields and quantum fields over 4-dimensional space-time. in Stochastic partial differential equations and applications, Ed. G. Da Prato, L. Tubaro, Pitman, Longman, 1992.

[AKu] Albeverio S. and Kusuoka S., A basic estimate for two-dimensional stochastic holonomy along Brownian bridges. J. Funct. Anal. 127 (1994), 132-154.

[AT] Albeverio S. and Tamura H., On the propagator of a scalar field in the presence of confining nonlinear electromagnetic force. preprint.

[B] Billingsley P., Ergodic theory and information. John Wiley and Sons, New York, 1965.

[CL] Carmona R. and Lacroix J., Spectral theory of random Schrödinger operators. Birkhäuser, Boston, 1990.

[F] Fukushima M., Dirichlet forms and Markov processes. North-Holland, Amsterdam, 1980.

[GJ] Glimm J. and Jaffe A., Quantum physics. 2nd ed. Springer-Verlag, Berlin, 1987.

[GV] Gel'fand I.M. and Vilenkin N. Ya., Generalized functions. Vol. 4, Academic Press, New York, 1964.

[Hi] Hida T., Brownian motion. Springer-Verlag, Berlin, 1980.

[I] Iwata K., The inverse of a local operator preserves the Markov property. Ann. 
Scuola Norm. Sup. Pisa 19 (1992), 223-253.

[KS] Karatzas I. and Shreve S.E., Brownian motion and stochastic calculus. SpringerVerlag, Berlin, 1987.

[Na] Nakao S., On the spectral distribution of the Schrödinger operator with random potential. Japan. J. Math. 3 (1977), 111-139.

[R] Rothe H.J., Lattice gauge theories, An introduction. World Scientific, Singapore, 1992.

[Si] Simon B., Functional integration and quantum physics. Academic Press, New York, 1979.

[St] Stein E.M., Singular integrals and differentiability properties of functions. Princeton, New Jersey, 1970.

[Ta] Tamura H., On the possibility of confinement caused by nonlinear electromagnetic interaction. J. Math. Phys. 32 (1991), 897-904.

[U] Ueki N., On spectra of random Schrödinger operators with magnetic fields. J. Math. 31 (1994), 177-187.

[Wi] Wilson K., Confinement of quarks. Phys. Rev. D, 10 (1974), 2445-2459.

Department of Mathematics

Faculty of Sciences

Kanazawa University

Kakuma-machi, Kanazawa 920-11

Japan

E-mail: nakane@kappa.s.kanazawa-u.ac.jp 